\title{
Jumne Seuroscience
}

The Official Journal of

August 1, 2000

the Society for Neuroscience

Volume 20 Number 15

Rapid Communications are available online only.

Rapid Communications (http://www.jneurosci.org)

RC87(1-5) Fractalkine Cleavage from Neuronal Membranes Represents an Acute Event in the Inflammatory Response to Excitotoxic Brain Damage

Gayle A. Chapman, Kitty Moores, David Harrison, Colin A. Campbell, Brian R. Stewart, and Paul J. L. M. Strijbos

RC88(1-4) Laminar Specificity of Local Circuits in Barrel Cortex of Ephrin-A5 Knockout Mice N. Harumi Yabuta, Amy K. Butler, and Edward M. Callaway

RC89(1-5) Glutamate Transmission in the Nucleus Accumbens Mediates Relapse in Cocaine Addiction Jennifer L. Cornish and Peter W. Kalivas

RC90(1-5) Mechanical Allodynia Caused by Intraplantar Injection of P2X Receptor Agonist in Rats: Involvement of Heteromeric P2 $\mathrm{X}_{2 / 3}$ Receptor Signaling in Capsaicin-Insensitive Primary Afferent Neurons

Makoto Tsuda, Schuichi Koizumi, Aya Kita, Yukari Shigemoto, Shinya Ueno, and Kazuhide Inoue

\section{Articles}

\section{Cellular/Molecular}

5575 Amphetamine Blocks Long-Term Synaptic Depression in the Ventral Tegmental Area Susan Jones, Johanna L. Kornblum, and Julie A. Kauer

5581 Modulation of Long-Term Depression by Dopamine in the Mesolimbic System Mark J. Thomas, Robert C. Malenka, and Antonello Bonci

5587 Complete and Long-Term Rescue of Lesioned Adult Motoneurons by Lentiviral-Mediated Expression of Glial Cell Line-Derived Neurotrophic Factor in the Facial Nucleus Andreas F. Hottinger, Mimoun Azzouz, Nicole Déglon, Patrick Aebischer, and Anne D. Zurn

5594 Efficacy and Stability of Quantal GABA Release at a Hippocampal Interneuron-Principal Neuron Synapse

Udo Kraushaar and Peter Jonas

5608 Brain Localization and Behavioral Impact of the G-Protein-Gated $\mathrm{K}^{+}$Channel Subunit GIRK4

Kevin Wickman, Christine Karschin, Andreas Karschin, Marina R. Picciotto, and David E. Clapham

5616 Developmental Regulation of Neuronal $\mathrm{K}_{\mathrm{Ca}}$ Channels by TGF $\beta 1$ : Transcriptional and Posttranscriptional Effects Mediated by Erk MAP Kinase

Loic Lhuillier and Stuart E. Dryer

5623 A Voltage-Independent Calcium Current Inhibitory Pathway Activated by Muscarinic Agonists in Rat Sympathetic Neurons Requires Both $\mathrm{G} \alpha_{\mathrm{q} / 11}$ and $\mathrm{G} \beta \gamma$

Paul J. Kammermeier, Victor Ruiz-Velasco, and Stephen R. Ikeda 
The Dopamine/D1 Receptor Mediates the Phosphorylation and Inactivation of the Protein Tyrosine Phosphatase STEP via a PKA-Dependent Pathway

Surojit Paul, Gretchen L. Snyder, Hisayuki Yokakura, Marina R. Picciotto, Angus C. Nairn, and Paul J. Lombroso

Facilitation of Recovery from Inactivation by External $\mathrm{Na}^{+}$and Location of the Activation Gate in Neuronal $\mathrm{Na}^{+}$Channels

Chung-Chin Kuo and Shu-Yuan Liao

Fos-Related Antigen 2: Potential Mediator of the Transcriptional Activation in Rat Adrenal Medulla Evoked by Repeated Immobilization Stress

Bistra B. Nankova, Mark Rivkin, Max Kelz, Eric J. Nestler, and Esther L. Sabban

Reduced Voltage Sensitivity of Activation of P/Q-Type $\mathrm{Ca}^{2+}$ Channels is Associated with the Ataxic Mouse Mutation Rolling Nagoya $\left(\operatorname{tg}^{\text {rol }}\right)$

Yasuo Mori, Minoru Wakamori, Sen-ichi Oda, Colin F. Fletcher, Naomi Sekiguchi, Emiko Mori, Neal G. Copeland, Nancy A. Jenkins, Kaori Matsushita, Zenjiro Matsuyama, and Keiji Imoto

5663 cAMP-Dependent Protein Kinase Inhibits mGluR2 Coupling to G-Proteins by Direct Receptor Phosphorylation

Hervé Schaffhauser, Zhaohui Cai, Frantisek Hubalek, Thomas A. Macek, Jan Pohl, Thomas J. Murphy, and P. Jeffrey Conn

Cell Surface Trk Receptors Mediate NGF-Induced Survival While Internalized Receptors Regulate NGF-Induced Differentiation

Yan-zhen Zhang, Daniel B. Moheban, Bevil R. Conway, Anita Bhattacharyya, and Rosalind A. Segal

Erb and c-Kit Receptors Have Distinctive Patterns of Expression in Adult and Developing Taste Papillae and Taste Buds

Susan K. McLaughlin

5689 Viral Gene Transfer of Dominant-Negative Kv4 Construct Suppresses an $\mathrm{O}_{2}$-Sensitive $\mathrm{K}^{+}$ Current in Chemoreceptor Cells

M. Teresa Pérez-García, José Ramón López-López, Armenia M. Riesco, Uta C. Hoppe, Eduardo Marbán, Constancio González, and David C. Johns

5696 Clinical Mutations in the L1 Neural Cell Adhesion Molecule Affect Cell-Surface Expression Hugh D. Moulding, Robert L. Martuza, and Samuel D. Rabkin

5703 Insulin-Like Growth Factor-1 Inhibits Mature Oligodendrocyte Apoptosis during Primary Demyelination

J. L. Mason, P. Ye, K. Suzuki, A. J. D'Ercole, and G. K. Matsushima

5709 Ibuprofen Suppresses Plaque Pathology and Inflammation in a Mouse Model for Alzheimer's Disease

G. P. Lim, F. Yang, T. Chu, P. Chen, W. Beech, B. Teter, T. Tran, O. Ubeda, K. Hsiao Ashe,

S. A. Frautschy, and G. M. Cole

5715 Delayed Mitochondrial Dysfunction in Excitotoxic Neuron Death: Cytochrome $c$ Release and a Secondary Increase in Superoxide Production

C. Marc Luetjens, Nguyen Truc Bui, Bernd Sengpiel, Gudrun Münstermann, Monika Poppe, Aaron J. Krohn, Elke Bauerbach, Josef Krieglstein, and Jochen H. M. Prehn

5724 The SNARE Vtila- $\beta$ Is Localized to Small Synaptic Vesicles and Participates in a Novel SNARE Complex

Wolfram Antonin, Dietmar Riedel, and Gabriele Fischer von Mollard

5733 Genetic Inactivation of an Inwardly Rectifying Potassium Channel (Kir4.1 Subunit) in Mice: Phenotypic Impact in Retina

Paulo Kofuji, Paul Ceelen, Kathleen R. Zahs, Leslie W. Surbeck, Henry A. Lester, and Eric A. Newman

5741 Induction of Postnatal Schwann Cell Death by the Low-Affinity Neurotrophin Receptor In Vitro and after Axotomy

Daniel E. Syroid, Peter J. Maycox, Merja Soilu-Hänninen, Steven Petratos, Tamara Bucci,

Patrick Burrola, Simon Murray, Surindar Cheema, Kuo-Fen Lee, Greg Lemke, and

Trevor J. Kilpatrick 
Keren Agam, Mark von Campenhausen, Simon Levy, Hagit Cohen Ben-Ami, Boaz Cook, Kuno Kirschfeld, and Baruch Minke

A Role for p27/Kip1 in the Control of Cerebellar Granule Cell Precursor Proliferation Kazuhiro Miyazawa, Toshiyuki Himi, Veronica Garcia, Hisakazu Yamagishi, Shigeaki Sato, and Yasuki Ishizaki

\section{Development/Plasticity/Repair}

5764 Differential Modulation of Proliferation in the Neocortical Ventricular and Subventricular Zones

Tarik F. Haydar, Feng Wang, Michael L. Schwartz, and Pasko Rakic

5775 BDNF Protects the Neonatal Brain from Hypoxic-Ischemic Injury In Vivo via the ERK Pathway

Byung Hee Han and David M. Holtzman

Depletion of a Microtubule-Associated Motor Protein Induces the Loss of Dendritic Identity Wenqian Yu, Crist Cook, Carley Sauter, Ryoko Kuriyama, Paul L. Kaplan, and Peter W. Baas

5792 Netrin-1 Promotes Thalamic Axon Growth and Is Required for Proper Development of the Thalamocortical Projection

Janet E. Braisted, Susan M. Catalano, Robert Stimac, Timothy E. Kennedy, Marc Tessier-Lavigne, Carla J. Shatz, and Dennis D. M. O'Leary

5802 Neocortical Origin and Tangential Migration of Guidepost Neurons in the Lateral Olfactory Tract

Naomi Tomioka, Noriko Osumi, Yasufumi Sato, Takayoshi Inoue, Shun Nakamura, Hajime Fujisawa, and Tatsumi Hirata

5813 Developmental Regulation of a Local Positive Autocontrol of Supraoptic Neurons Vivien Chevaleyre, Govindan Dayanithi, Françoise C. Moos, and Michel G. Desarménien

Long-Lasting Inhibitory Synaptic Depression is Age- and Calcium-Dependent Vibhakar C. Kotak and Dan H. Sanes

\section{Behavioral/Systems}

Prominence of Direct Entorhinal-CA1 Pathway Activation in Sensorimotor and Cognitive Tasks Revealed by 2-DG Functional Mapping in Nonhuman Primate Elżbieta Sybirska, Lila Davachi, and Patricia S. Goldman-Rakic

5835 Eye Position Signal Modulates a Human Parietal Pointing Region during Memory-Guided Movements

Joseph F. X. DeSouza, Sean P. Dukelow, Joseph S. Gati, Ravi S. Menon, Richard A. Andersen, and Tutis Vilis

5841 Malformation of the Functional Organization of Somatosensory Cortex in Adult Ephrin-A5 Knock-Out Mice Revealed by In Vivo Functional Imaging Neal Prakash, Pierre Vanderhaeghen, Susana Cohen-Cory, Jonas Frisén, John G. Flanagan, and Ron D. Frostig

5848 Rescue of Locomotor Impairment in Dopamine D2 Receptor-Deficient Mice by an Adenosine A2A Receptor Antagonist Shiro Aoyama, Hiroshi Kase, and Emiliana Borrelli

5853 Remote Episodic Memory Deficits in Patients with Unilateral Temporal Lobe Epilepsy and Excisions Indre V. Viskontas, Mary Pat McAndrews, and Morris Moscovitch

5858 Role of Inspiratory Pacemaker Neurons in Mediating the Hypoxic Response of the Respiratory Network In Vitro

Muriel Thoby-Brisson and Jan-Marino Ramirez

5867 Nonphotic Entrainment by 5-HT $\mathrm{HT}_{1 \mathrm{~A} / 7}$ Receptor Agonists Accompanied by Reduced Per1 and Per2 mRNA Levels in the Suprachiasmatic Nuclei Kazumasa Horikawa, Shin-ichi Yokota, Kazuyuki Fuji, Masashi Akiyama, Takahiro Moriya, Hitoshi Okamura, and Shigenobu Shibata 

of Action

S. K. Joshi, Xin Su, Frank Porreca, and G. F. Gebhart

5880 Selection of Currently Relevant Memories by the Human Posterior Medial Orbitofrontal Cortex Armin Schnider, Valerie Treyer, and Alfred Buck

Occlusion and the Interpretation of Visual Motion: Perceptual and Neuronal Effects of Context

Robert O. Duncan, Thomas D. Albright, and Gene R. Stoner

Errata: In the legend to the cover picture appearing in the July 1, 2000 issue, the following article information was omitted. The cover picture is from the article "Retinal Ganglion Cell Axon Guidance in the Mouse Optic Chiasm: Expression and Function of Robos and Slits," by Lynda Erskine, Scott E. Williams, Katja Brose, Thomas Kidd, Rivka A. Rachel, Corey S. Goodman, Mark Tessier-Lavigne, and Carol A. Mason, appearing on pages 4975-4982 of the issue. In addition, it should be noted that this article was published in conjunction with two related articles, also in the July 1, 2000 issue: "Slit2 Is a Repellent for Retinal Ganglion Cell Axons," by Simone P. Niclou, Li Jia, and Jonathan A. Raper (pages 4962-4974), and "Slit Inhibition and Retinal Axon Growth and Its Role in Retinal Axon Pathfinding and Innervation Patterns in the Diencephalon," by Thomas Ringstedt, Janet E. Braisted, Katja Brose, Thomas Kidd, Corey Goodman, Mark Tessier-Lavigne, and Dennis D. M. O'Leary (pages 4983-4991). In the legend to the cover picture appearing in the July 15, 2000 issue, the following article information was omitted. The cover picture is from the article "Selective Alterations in GABA $\mathrm{A}$ Receptor Subtypes in Human Temporal Lobe Epilepsy," by Fabienne Loup, Heinz-Gregor Wieser, Yasuhiro Yonekawa, Adriano Aguzzi, and Jean-Marc Fritschy, appearing on pages 5401-5419.

In the companion articles "Different Subthreshold Mechanisms Underlie Song Selectivity in Identified HVc Neurons of the Zebra Finch," by Richard Mooney, and "Intrinsic and Extrinsic Contributions to Auditory Selectivity in a Song Nucleus Critical for Vocal Plasticity," by Merri J. Rosen and Richard Mooney, which appeared in the July 15, 2000 issue, page numbers for the corresponding companion article were omitted in the reference list of each article and are as follows. In the article by Mooney, the page numbers for the citation for Rosen and Mooney should read 5437-5448. In the article by Rosen and Mooney, the page numbers for the citation for Mooney should read 5420-5436.

Cover picture: Kir4.1 potassium channel expression in glial cells of the retina. Astrocytes are shown in blue, Müller cells in red, and Kir4.1 channels in green in this triple-labeled confocal fluorescence image of the rat retina. The potassium channel is prominently expressed in Müller cell processes along blood vessels (bottom left) but not in astrocytes. Overlap of Kir4.1 channels and Müller cells is shown in yellow. The black circles are unlabeled ganglion cell somata, which are surrounded by Müller cell processes. Antibodies against glial fibrillary acidic protein (astrocytes), glutamine synthetase (Müller cells), and Kir4.1 were used. For details, see the article by Kofuji et al., in this issue (pages 5733-5740).

Persons interested in becoming members of the Society for Neuroscience should contact the Membership Department, Society for Neuroscience, 11 Dupont Circle, NW, Suite 500, Washington, DC 20036, phone 202-462-6688.

Instructions for Authors appear at the end of the January 1, 2000 issue. Copies of the Instructions can be obtained by writing to The Journal of Neuroscience, Society for Neuroscience, 11 Dupont Circle, NW, Suite 500, Washington, DC 20036, phone 202-4626688, fax 202-462-1547, e-mail jn@sfn.org. The Instructions are also available via Internet (http://www.jneurosci.org/misc/itoa.shtml). Rapid Communications Instructions for Authors appear at the end of the January 15, 1999 issue and are also available via Internet (http://www.sfn.org/RapidComm/ifa.html). Submissions should be sent to the above address. Scientific inquiries concerning manuscripts can be made directly to Dr. Gordon M. Shepherd, Editor-in-Chief, The Journal of Neuroscience, Section of Neurobiology, Yale University School of Medicine, 333 Cedar Street, New Haven, CT 06510, phone 203-785-4336, fax 203-785-6990, e-mail jneurosci@yale.edu.

The Journal of Neuroscience requests that authors send a disk containing an electronic file of their manuscript with each submission. Most word processing software can be used; see the Instructions for Authors for detailed guidelines on acceptable disk and file formats. 